\title{
MAiD in the Netherlands led by physicians
}

$\mathrm{F}$ rom the beginning, Medical Aid in Dying (MAiD) in the Netherlands was doctor led and its practice rooted in concern for protecting the physician. The result is a nationwide system of expert consultants delivering MAiD and euthanasia review committees making sure it is done properly.

Canada would do well to adopt some of these protocols, Dr. Gerrit Kimsma, a family physician and philosophy professor at Radboud University Nijmegan Medical Center in the Netherlands, advised at the recent Ottawa Conference on MAiD.

The Dutch law was initiated by the medical profession, "instead of being confrontational as it is so clearly in your country," Kimsma said.

Canada's MAiD legislation arose from the 2015 Carter case in the Supreme Court, in which Kimsma served as an expert witness. Bill C-14, which became law on June 17, removed prohibitions against assisted dying and established national guidelines while sparking uncertainty among health professionals on how exactly to proceed.

In the Netherlands, discussions began in 1984 with doctors wanting to protect oncologists who were providing MAiD to suffering patients who had no other medical recourse. "The medical system instigated it, but the judicial system supported it," said Kimsma.

The rules and regulations in the 2002 Dutch Euthanasia law "were largely worked out by the medical profession ... with a focus on ethics and medical science," Kimsma told attendees at the University of Ottawa Centre for Health Law, Policy and Ethics event. The law is physician centred, he maintained.

The result has been widespread acceptance, he added. When initially discussed, only $20 \%-30 \%$ of Dutch physicians supported MAiD. Now that figure is $90 \%$ "because the practice developed in a supported way and physicians feel supported by it."

In Canada, MAiD protocols include having two independent witnesses sign requests, two medical opinions agree that the patient meets criteria and a 10-day waiting period between the request and MAiD.
Kimsma elaborated on three safeguards in the Netherlands that protect the physician.

First is legal protection. If the physician acts in accordance with criteria of due care there will not be legal repercussions, Kimsma emphasized. These criteria require the patient to be fully informed, aware and communicative, and experiencing unbearable and hopeless suffering. The physician must also consult with another physician and use a prescribed protocol in ending life. In addition, there must be an absence of reasonable alternatives for both physician and patient.

"This is the relational aspect, whereas ... your law focuses on the patient," said Kimsma who practises MAiD. "I often feel there are medical options. I argue with my patients until we have agreement. If I'm going to do it, I have to be sure."

Physicians refuse MAiD in twothirds of cases, he said.

The second safeguard is a cadre of some 600 specially trained Support and Consultation on Euthanasia in The Netherlands (SCEN) consultants. "We found in practice a lot of variation," in how MAiD was carried out, explained Kismsa. "You need special training and you need to do it repeatedly in order to do it right."

"That is something that is highly valuable and you don't get that in Canada. You should think about organizing it."

SCEN consultants are available on short notice (within 48 hours), they meet with the family and patient, and with the patient alone, examine the records, and ensure the law is met and the doctor protected. The consultants are organized in regional groups and meet at least four times annually but communicate regularly.

"This is a procedure that is really flexible but also fast," said Kimsma, who is a SCEN consultant and trainer. The 10-day waiting period in Canada may be problematic, he added. There is a protocol for sidestepping the requirement, "but that's another step. You're not really making it easy for your physicians. If I were a Canadian physician I would be irritated."

The third safeguard occurs after the intervention. All the information is sent to a regional Euthanasia Review Committee (ERC) comprised of a lawyer, physician and ethicist who meet monthly to review cases. Kimsma was on such a committee for a decade.

The committee determines that the law was followed. Sometimes physicians are asked to provide more information.

"I've been on both sides and I'm impressed with how much the committee was actually siding with the physicians and understands what the physician did," said Kimsma.

MAiD was politically charged in the Netherlands, as it was in Canada. This was in part fuelled by misinformation, said Kimsma. In 1991, for example, some groups claimed there were 50000 physician-assisted deaths per year; no one knew for sure. The Dutch partly depoliticized the issue by forming the Research Committee on Medical Practice on Euthanasia to gather data. In 1991, it found there were 1800 cases of MAiD (2.9\% of all deaths).

The committee has continued to gather these data every five years; in 2015, there were 5516 cases of MAiD (3.9\% of all deaths). Of those cases, 109 patients suffered dementia, 56 were psychiatric patients and 183 had multiple ailments.

"The amount of data and the effects of the data are clearly undeniably positive," said Kimsma. "This is what you do not have in Canada."

Canadian law stipulates that every province has to do surveillance, but so far only Quebec is complying, perhaps because its law came into effect seven months before the national legislation. But Quebec is having problems gathering data, said Isabelle Marcoux, an assistant professor in the Interdisciplinary School of Health Sciences at the University of Ottawa. The questionnaire is unclear and too detailed, she said.

Canadian research is underway to improve data generation. Marcoux and colleagues, for example, developed and piloted a questionnaire on different types of end-of-life practices. They are now starting a national project to assess the frequency of various endof-life practices and determine how decisions are made. 
Adopting aspects of the Dutch system may be problematic, Marcoux said. For example, Canada's low population density makes it difficult to provide timely services in remote areas.

There's also a difference in where people die: $66 \%$ of Canadians die in hospital compared with $40 \%$ of the Dutch. This may affect delivery of MAiD. And many Canadians don't enjoy long-standing relationships with their physician, like Dutch people typically have. "It's hard to make an assessment when you don't know the patient and their values," said Marcoux.
"Particularly in cases when intervention is urgent."

"The challenge will be to see the quality of MAiD you can reach," said Kimsma. "You will have to find your own way." - Barbara Sibbald, CMAJ

CMAJ 2016. DOI:10.1503/cmaj.109-5342

\section{Most contributors to major US cancer guidelines have financial conflicts}

$\mathrm{O}$ $f$ the 125 contributors to four major clinical guidelines for cancer treatment in the United States, $86 \%$ had at least one financial competing interest with industry, according to a recent study. This is of concern because doctors rely on these guidelines to make decisions about cancer treatments for their patients, and financial ties have the potential to bias a physician toward recommending a specific company's product, according to study coauthor Stacie Dusetzina, an assistant professor in the Division of Pharmaceutical Outcomes and Policy at the University of North Carolina Eshelman School of Pharmacy.

"If the recommended product is expensive and doesn't have clear evidence of benefiting patients, then it could just be adding more costs to the patient," Dusetzina said in an email to CMAJ. "These costs are referred to as 'financial toxicity' in cancer care in the US, as they can often be very high and a huge burden for patients."

The study, published in JAMA Oncology, found that $84 \%$ of guideline authors received general payments from industry (for things such as consulting, meals and lodging) and $47 \%$ received money for research. The average general payment had a value of US\$10 011; the average research payment was US\$236 066. The study considered financial competing interests in 2014 among contributors to four National Comprehensive Cancer Network (NCCN) guidelines: for lung, breast, prostate and colorectal cancer.

"The NCCN does a good job of managing conflicts of interest overall," said Dusetzina. "I think the most important thing is to be explicit about payments received so that any potential biases are recognized and recommen-

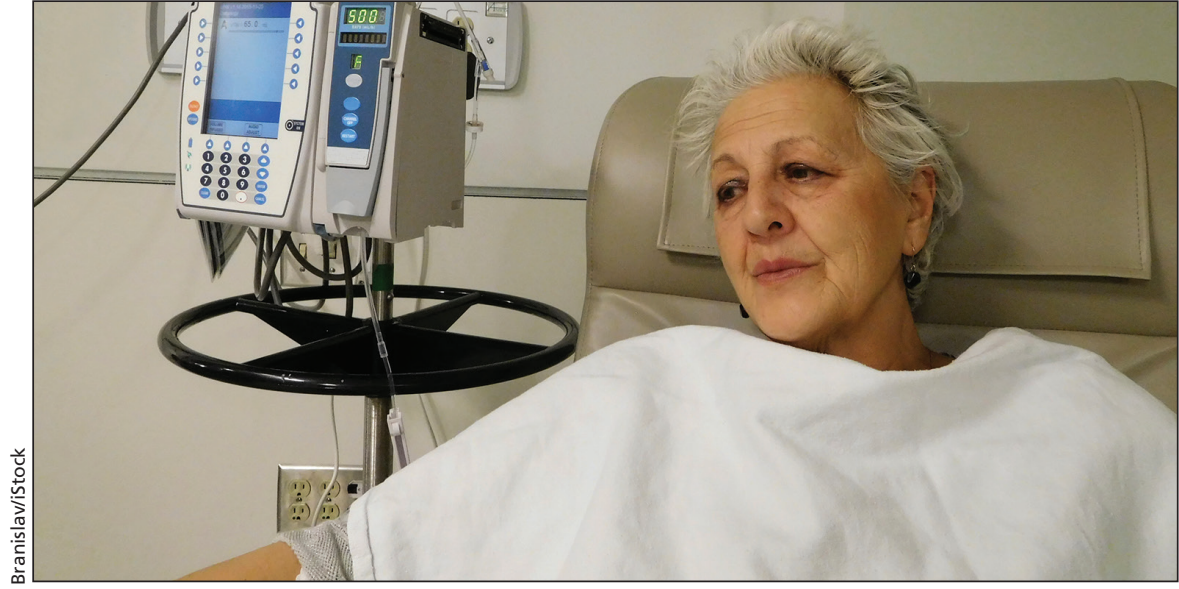

Many doctors rely on clinical guidelines to make decisions about cancer treatments.

dations are considered in light of these potential conflicts."

When contacted by CMAJ for comment, a representative for the National Comprehensive Cancer Network said it was not holding interviews at this time and was preparing a formal public response.

As pharmaceuticals get more and more expensive, clinical guidelines become all the more valuable in guiding treatment decisions, according to Dr. Otis Brawley, chief medical officer of the American Cancer Society. "The issue is that you have people who make money doing the treatments actually deciding what the treatments should be," he said "Unfortunately, these are also the people who are the most expert in outcomes."

The good thing about financial competing interests, however, is that they are easier than ever to monitor in the US, thanks to the 2010 Physician Payments Sunshine Act, a US law that requires drug companies to disclose payments to doctors. According to Brawley, the usual practice on guideline committees is to exclude individuals with major competing interests. Physicians with minor financial ties to industry must still disclose these, but can participate if they believe they can be objective and open-minded.

"The key is you can't avoid them; you need to manage them," Brawley said about financial competing interests.

Of more concern are what Brawley calls emotional competing interests. These occur when a member of a guideline committee absolutely refuses to reconsider his or her opinion on a treatment in light of new evidence, he said. Such stubborn individuals aren't practising evidence-based medicine, he said; they are practising faith-based medicine.

"Those ones are a bigger problem. The guys who are making money know they are making money," he said. "The close-minded individual doesn't realize he or she is prejudiced." - Roger Collier, CMAJ

CMAJ 2016. DOI:10.1503/cmaj.109-5334 